\title{
E-Mail Interviewing in Qualitative Research: A Methodological Discussion
}

\author{
Lokman I. Meho \\ School of Library and Information Science, Indiana University, 1320 E. 10th Street, \\ LI 011, Bloomington, IN 47405. E-mail: meho@indiana.edu
}

\begin{abstract}
This article summarizes findings from studies that employed electronic mail (e-mail) for conducting indepth interviewing. It discusses the benefits of, and the challenges associated with, using e-mail interviewing in qualitative research. The article concludes that while a mixed mode interviewing strategy should be considered when possible, e-mail interviewing can be in many cases a viable alternative to face-to-face and telephone interviewing. A list of recommendations for carrying out effective e-mail interviews is presented.
\end{abstract}

\section{Introduction}

The past two decades have seen a considerable increase in the number of studies in library and information science (LIS) that employ qualitative research methods. This increase has, in turn, resulted in a noticeable shift towards studies that rely on observation and in-depth (or less-structured) interviewing, as opposed to questionnaires or structured interviewing. The goal of both observation and in-depth interview methods is to improve understanding of social and cultural phenomena and processes rather than to produce objective facts about reality and make generalizations to given populations (Fidel, 1993; Pettigrew, Fidel, \& Bruce, 2001; Wang, 1999). Over the years, however, researchers have identified challenges associated with the observation and in-depth interview methods, including cost, time, and limited access to research participants (Denzin \& Lincoln, 2005; Gubrium \& Holstein, 2002; Kvale, 1996; Miles \& Huberman, 1994; Patton, 2002; Strauss \& Corbin, 1998; Taylor \& Bogdan, 1998). Challenged with the task of identifying new methods or tools for conducting more effective research while retaining or improving quality, researchers started to explore using the Internet for carrying out qualitative research.

These researchers began to use (and still do) three main types of Internet-based qualitative research methods: online synchronous interviews, online asynchronous interviews,

Received May 25, 2005; revised June 23, 2005; accepted August 3, 2005

(C) 2006 Wiley Periodicals, Inc. • Published online 25 May 2006 in Wiley InterScience (www.interscience.wiley.com). DOI: 10.1002/asi.20416 and virtual focus groups. ${ }^{1}$ In contrast to studies that used online synchronous interviews (Bowker \& Tuffin, 2004; Madge \& O'Connor, 2002; Mann \& Stewart, 2000) and those that used virtual focus groups (Burton \& Bruening, 2003; Chase \& Alvarez, 2000; Gaiser, 1997; Mann \& Stewart, 2000; Schneider, Kerwin, Frechtling, \& Vivari, 2002; Underhill \& Olmsted, 2003), studies that used online asynchronous interviewing have never been reviewed as a separate body of literature and their specific characteristics have nearly always been subsumed under the broader category of online research methods (e.g., Kraut et al., 2004; Madge \& O'Connor, 2004). To fill this gap, the current paper reviews studies that used online, asynchronous, in-depth interviewing within the context of qualitative research. In doing so, the article addresses two questions:

What opportunities, constraints, and challenges does online, asynchronous, in-depth interviewing present for collecting qualitative data?

How can in-depth e-mail interviews be conducted effectively?

Before discussing these questions, it is important to note that online, asynchronous, in-depth interviewing, which is usually conducted via e-mail, is, unlike e-mail surveys, semistructured in nature and involves multiple e-mail exchanges between the interviewer and interviewee over an extended period of time. Online, asynchronous, in-depth interviewing is also different from virtual focus groups in that the information volunteered by individual participants is not shared with, viewed, or influenced by other participants (Schneider et al., 2002). With the exception of Meho and Tibbo (2003), LIS researchers have yet to adopt this method of interviewing in their qualitative research. Exploring the value of e-mail interviewing in qualitative research and

\footnotetext{
${ }^{1}$ Electronic questionnaires (via Web page delivery or electronic mail) are among the earliest and most popular online methods used by researchers. These, however, are considered quantitative in nature and the studies based on them are not reviewed here. For excellent reviews of online surveys or questionnaires, see Birnbaum (2004), Couper (2000), Dillman (2000), and Tourangeau (2004).
} 
knowing under what conditions it can be most effective and how to implement it, should be useful to LIS researchers. This knowledge could be particularly useful to those who study people who prefer to be interviewed online rather than face-to-face, as well as people who are not easily accessible or are geographically far apart.

What follows is a review of studies that employed qualitative e-mail interviewing, a summary of their major findings, and a list of recommendations for carrying out effective e-mail interviews. $^{2}$

\section{Review of the Literature}

Citation and bibliographic searches using multiple types of sources and strategies indicate that the use of in-depth, e-mail interviewing is rapidly increasing. In 2003 and 2004 alone, for example, there were as many qualitative studies using this data collection method as in all previous years (see below). Moreover, nearly all of the studies conducted before 2003 were methodological in nature, aiming simply to test the suitability of e-mail for qualitative interviewing. In contrast, most of the studies conducted since 2003 have not addressed methodological issues; this suggests that e-mail interviewing has become a viable tool for qualitative research.

\section{Methodological Studies}

In the earliest study Foster (1994) used e-mail for conducting qualitative interviews with subscribers to a listserv. His intentions were both to study the ways in which university instructors conducted curriculum planning and to explore the advantages of e-mail interviewing, along with the challenges that could arise were the method not employed carefully. Murray $(1995,1996)$ interviewed five nurses to study why and how they used computer-mediated communication and to examine the potentials of e-mail for interviewing research participants. Persichitte, Young, and Tharp (1997) interviewed six education professionals by e-mail to examine how they used technology at work and to develop guidelines for conducting e-mail interviews (see also Young, Persichitte, \& Tharp, 1998). Murray and Sixsmith (1998) conducted electronic interviews with 21 prosthesis users located in Australia, Canada, the Netherlands, United Kingdom, and United States in order to explore the viability of e-mail as a qualitative research medium for in-depth interviewing. After educating her third- and fourth-year college students about qualitative methods and in-depth interviewing, Curasi (2001) asked them to interview 48 consumers by e-mail and face-to-face to examine how data from the two types of interviews compare and how to increase the effectiveness of the e-mail method.

\footnotetext{
${ }^{2}$ The relevant literatures of discourse analysis, content analysis, and computer-mediated communication (CMC) are not reviewed here. Discourse analysis is described in detail in Dijk (1997) and Schiffrin, Tannen, and Hamilton (2001); content analysis in Krippindorff (2004), Patton (2002), and Weber (1990); and CMC in Herring (2002), Knapp and Daly (2002), and Thurlow, Tomic, and Lengel (2004).
}

\section{Non-Methodological Studies}

Kennedy (2000) conducted in-depth, e-mail interviews with 17 women who are designers to determine the kinds of experiences they have on the Internet and the impact these experiences have on their Internet Web site activities, Internet personal activities, and non-Internet personal and/or social activities. Karchmer (2001) used e-mail interviewing to explore $13 \mathrm{~K}-12$ teachers' reports about how the Internet influenced literacy and literacy instruction in their classrooms. Meho and Tibbo (2003) used e-mail interviewing to explore and model the information-seeking behavior of social science faculty; they interviewed 60 scholars from 14 different countries (see also Meho, 2001). Kim, Brenner, Liang, and Asay (2003) used e-mail to interview ten 1.5Generation Asian American college students to study their adaptation experiences as immigrants and their current experiences as young adults. Hodgson (2004) used e-mail to interview 22 self-reported self-injurers to learn about their stigma management techniques and their motives for selfinjury. Lehu (2004) conducted in-depth interviews with 53 top-level managers and advertising executives to investigate why brands age and what managers subsequently do to rejuvenate them. Murray (2004) interviewed 35 prosthesis users by e-mail and face-to-face to understand the embodied perceptual experience of successful prosthesis. Murray and Harrison (2004) conducted e-mail and face-to-face interviews with 10 stroke survivors to investigate the meaning and experience of being a survivor. Olivero and Lunt (2004) conducted semistructured long e-mail and face-to-face interviews with 28 adult Internet users to explore their views on privacy. More details about the above mentioned 14 studies are provided in Table 1.

Although only Curasi (2001), Meho and Tibbo (2003), Murray (2004), Murray and Harrison (2004), and Olivero and Lunt (2004) collected both e-mail and face-to-face interview data, the majority of the 14 studies summarized above discussed the benefits and challenges of e-mail interviewing in qualitative research and how to alleviate or eliminate some of those problems, or how to conduct more efficient and effective e-mail interviews. The following is a summary of their findings.

\section{Benefits and Challenges Associated With the Use of E-Mail Interviewing in Qualitative Research}

\section{Cost and Efficiency}

E-mail interviews cost considerably less to administer than telephone or face-to-face interviews. Researchers can invite participation of large or geographically dispersed samples of people by sending them e-mail messages individually or through listservs, message boards, or discussion groups, rather than making long-distance telephone calls, using regular mail, or traveling to the location of participants. The use of e-mail in research also decreases the cost of transcribing. Data from e-mail interviews are generated in 


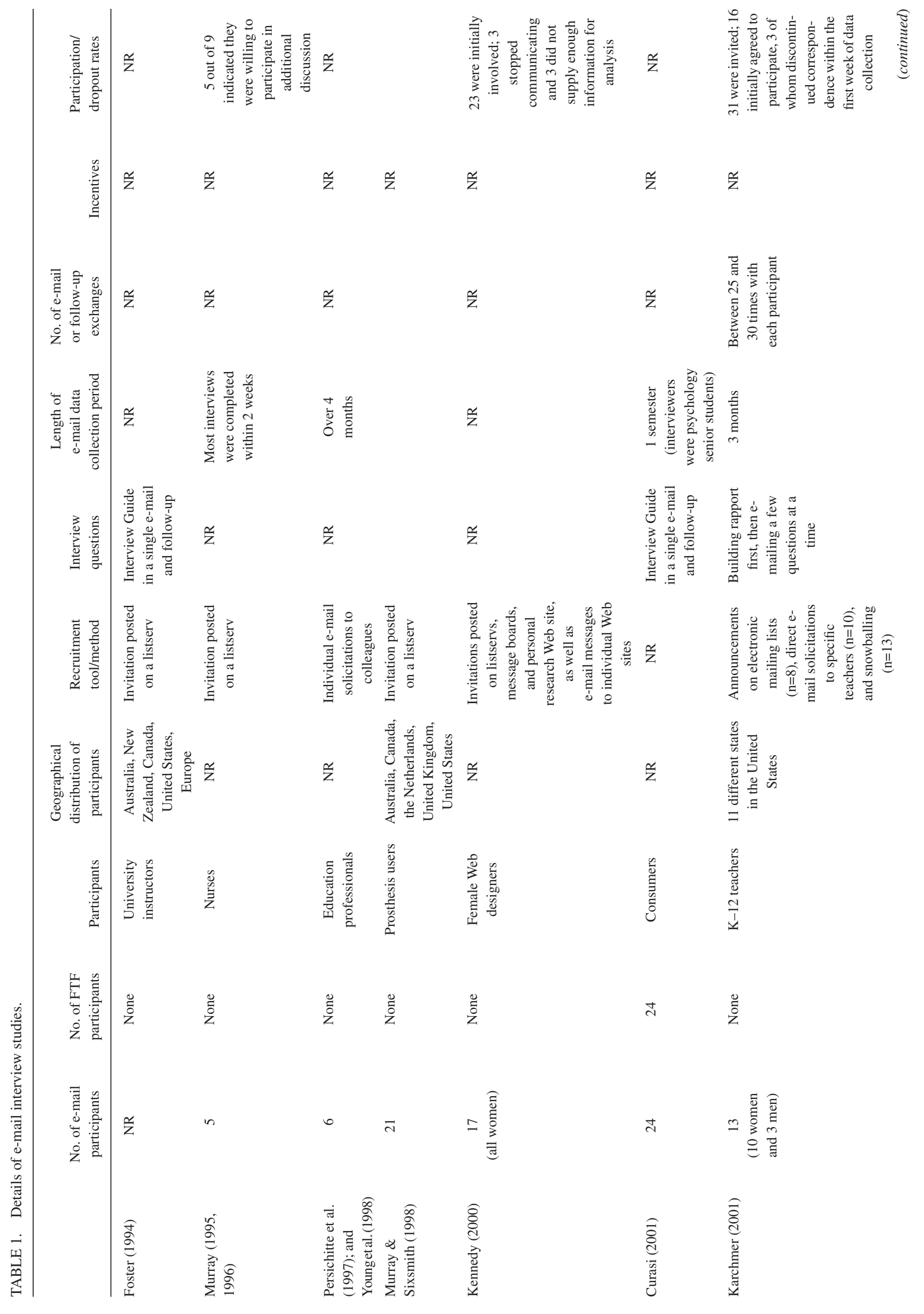




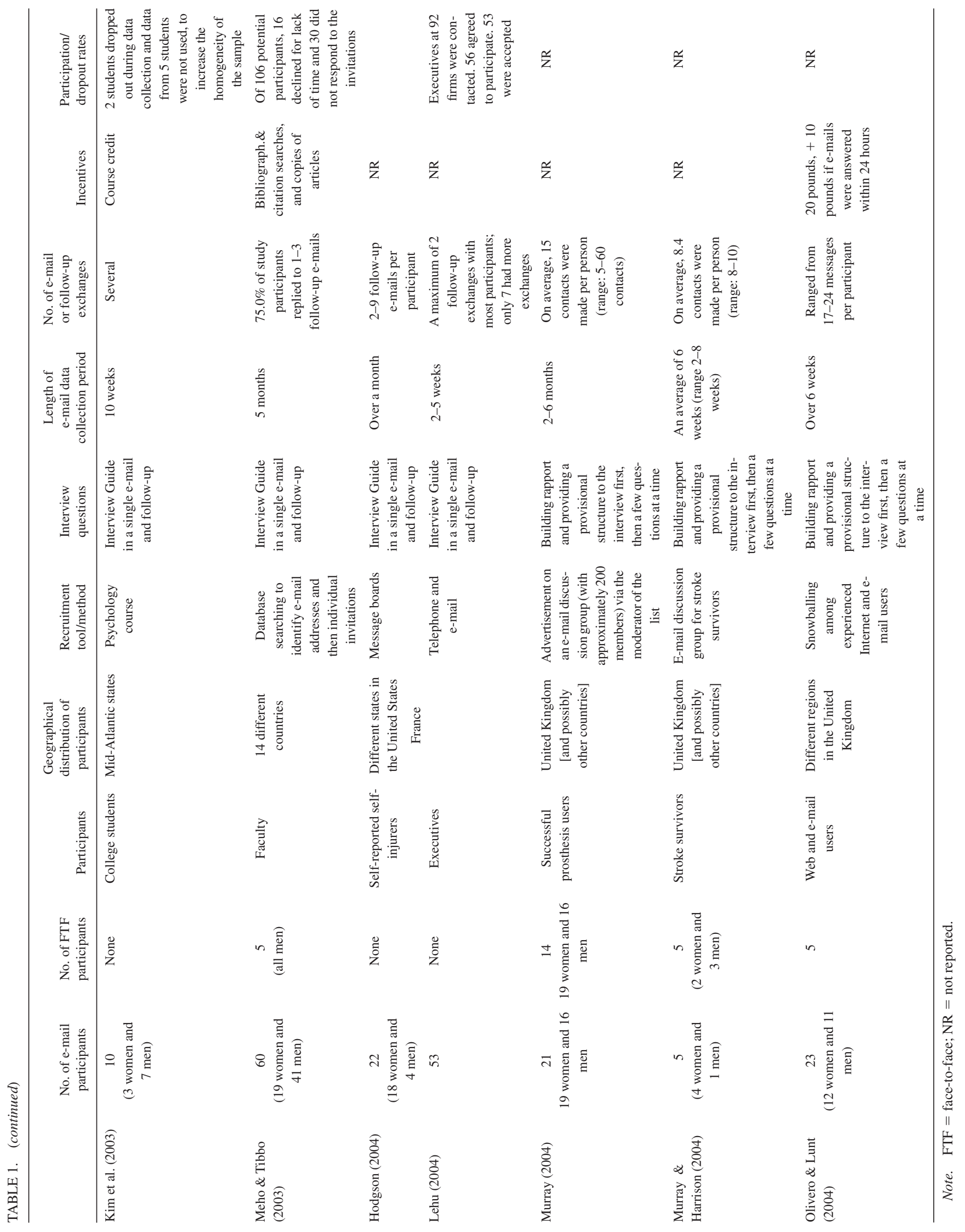


electronic format and require little editing or formatting before they are processed for analysis.

E-mail also eliminates the need for synchronous interview times and allows researchers to interview more than 1 participant at a time, because a standard interview schedule or list of questions can be sent individually to several participants at once, irrespective of their geographical location or time zone. However, the time period required to collect e-mail interview data varies from one study to another. Some researchers report a delay of several months before data collection is complete, while others wait only a week. This variation occurs because it may take days or even weeks before a respondent replies to an e-mail message. The number of follow-up exchanges between the researcher and participants may also fluctuate greatly; some interviewers complete data collection after only one follow-up exchange, whereas others may require more than 30 exchanges (see Table 1).

Overall, the length of the data collection period depends on several factors, including but not limited to: the number of participants in each study, the number of questions asked, the degree of commitment or motivation of the participants, the quantity and quality of data gathered, the time both the participants and the interviewers can afford to spend on these interviews, and access to the Internet at the time questions were e-mailed. Some studies showed that when online communication was stretched over a long period of time, participants experienced a degree of affirmation for their participation (Bowker \& Tuffin, 2004; Walther, 1996). As discussed further below, however, other studies show that the longer it takes to complete an interview with a participant, the higher the possibility of dropouts or frustration to both the researcher and the interviewees (Hodgson, 2004). Methods or procedures that a researcher could employ to reduce the possibilities of dropouts or frustration caused by length or number of interviews are discussed below.

\section{Democratization and Internationalization of Research}

Although e-mail interviewing limits the research to those people with access to the Internet, the method, on the other hand, democratizes and internationalizes research. In contrast to face-to-face and telephone interviewing, e-mail interviewing enables researchers to study individuals or groups with special characteristics or those often difficult or impossible to reach or interview face-to-face or via telephone, such as executives (Lehu, 2004), prosthesis users (Murray, 2004; Murray \& Sixsmith, 1998), self-reported self-injurers (Hodgson, 2004), stroke survivors (Murray \& Harrison, 2004), and people with disabilities (Bowker \& Tuffin, 2004), or those who are geographically dispersed (Foster, 1994; Hodgson, 2004; Karchmer, 2001; Meho \& Tibbo, 2003; Murray, 2004; Murray \& Harrison, 2004; Murray \& Sixsmith, 1998; Olivero \& Lunt, 2004) or located in dangerous or politically sensitive sites (Meho \& Tibbo, 2003). Moreover, e-mail enables the interviewing of shy people or people who do not or cannot express themselves as well in talking as they do in writing, especially when the language used in communicating with participants is their second one (Karchmer, 2001; Kim et al., 2003). In short, e-mail allows the researcher to interview groups or communities that would not and could not have been studied otherwise.

\section{Sample Recruitment}

Recruiting in e-mail interviewing studies is done in multiple ways, including individual solicitations, snowballing, or invitations through listservs, message boards, discussion groups, or personal research Web sites. Because these are the same methods employed by online survey researchers, it was not surprising that e-mail interviewing researchers face similar problems in recruiting participants. For example, although recruiting is easy in some cases, in others it can be daunting because even when e-mail addresses are found or invitations are sent to listservs and message boards, not all potential participants read the invitations (Meho \& Tiboo, 2003). The number of qualitative studies that have employed e-mail interviewing is insufficient for making generalizations, but experience with online survey research indicates that, due to information overload, many people delete invitations before they are read. To ensure sufficient participation, researchers who encounter high undeliverable rates usually send reminders to those who did not reply to initial invitations. As with traditional mail and e-mail surveys, reminders can significantly increase participation rates (see Meho \& Tibbo).

A number of the e-mail interviewing studies reviewed here also share findings similar to those of online survey research in terms of high rates of nondelivery (Dommeyer \& Moriarty, 2000; Frost, 1998; Meho \& Tibbo, 2003; Oppermann, 1995). Among other reasons, this occurs because people change or lose their e-mail addresses (e.g., students who graduate from school, faculty members who change jobs, or people who change their Internet service providers). But because a representative sample is not a goal in qualitative research, authors who employ e-mail interviewing usually overcome this problem of high nondelivery rate by inviting new or additional individuals to participate, if needed.

\section{Informed Consent and Confidentiality}

As in conventional studies, researchers who employ qualitative e-mail interviewing develop informed consent, providing participants detailed information about the research in which they are asked to participate and ensuring that they understand fully what participation would entail, including any possible risks. Participants in e-mail interview research are asked to take part in a study only after they provide their consent, which can be given to the researcher in a number of ways, including but not limited to: returning via fax or snail mail a signed form that was sent as an e-mail attachment, e-mailing back a signed form, or simply replying via e-mail affirmatively to an invitation to participate by stating in the message that the consent form was read and agreed to. The 
right to withdraw from a study at any time is also included in the consent form. For more details about informed consent in online research, see Kraut et al. (2004).

According to Kraut et al. (2004), "research on the Internet is not inherently more difficult to conduct or inherently riskier to subjects than more traditional research styles. But because the Internet is a relatively new medium for conducting research, it raises ambiguities that have been long settled in more conventional laboratory and field settings" (p. 114). In addressing these issues, researchers and Institutional Review Boards (IRBs) will need expertise, which many currently lack. ${ }^{3}$ This includes expertise about both online behavior and technology. For example, security, digital signatures, procedures for stripping identifying information, and provisions for one-on-one debriefing require specialized technical expertise. As in the case of face-to-face research, in the context of e-mail interviewing researchers need to ensure that adequate provisions are taken to protect the privacy of participants and to maintain the confidentiality of data. This is so because identifying information such as records of statements, attitudes, or behaviors, coupled with names, email addresses, partially disguised pseudonyms, or other identifying information, may be inadvertently disclosed either when the data are being collected or, more commonly, when they are stored on a networked computer connected to the public Internet (Kraut et al., 2004; Singer \& Levine, 2003). Emphasizing to participants that certain measures will be adopted to maximize confidentiality is necessary. Examples of these measures include the use of pseudonyms and hiding the user names, domain names, and any other personal identifiers when publishing or storing interview data.

Many people perceive online communication as anonymous because there is no in-person contact and thus, little accountability. This anonymity may explain why some people are more willing to participate in e-mail interview studies, whereas others are more willing to stop participating, not respond in a timely fashion, embellish more, or be less friendly to the interviewer (Hodgson, 2004; Mann \& Stewart, 2000). As explained further below, the anonymity afforded by online communication can be an important factor in increasing self-disclosure (Herring, 1996; Mann \& Stewart; Tidwell \& Walther, 2002) and in facilitating a closer connection with interviewees' personal feelings, beliefs, and values (Matheson, 1992). The American Psychological Association (Kraut et al., 2004), the American Association for the Advancement of Science (Frankel \& Siang, 1999), the Association of Internet Research (Ess, 2002), Brownlow and O'Dell (2002), Eysenbach and Till (2001), Mann and Stewart (2000), Pittenger (2003), and Sharf (1999) all provide excellent detailed discussion on ethical issues relevant to online research.

\footnotetext{
${ }^{3}$ The American Psychological Association (APA) recommends that all IRB boards have technical consultants who can be called on to resolve these issues when needed. APA further recommends that IRBs undertake an educational mission to inform researchers about the issues, the judgments that are now involved, and remedies for ensuring the health and protection of subjects in online research (Kraut et al., 2004).
}

\section{Medium Effects}

One of the most important differences between e-mail interviews and face-to-face or telephone interviews involves media richness, that is, the ability of a communication medium to foster interaction and feedback and to permit people to communicate with many kinds of cues, using multiple senses (Panteli, 2002; Robert \& Dennis, 2005). Having said this, face-to-face interviews are then expected to provide richer data than telephone interviews and telephone interviews are expected to provide richer data than e-mail interviews (Schneider et al., 2002). This is true because in e-mail interviews, for example, the interviewer will not be able to read facial expressions and body language, make eye contact, or hear voice tones of the participants. As a result, it is possible that some important visual or nonverbal cues are missed online that would be observed during face-to-face data collection (Selwyn \& Robson, 1998).

On the other hand, e-mail interviews reduce, if not eliminate, some of the problems associated with telephone or face-to-face interviews, such as the interviewer/interviewee effects that might result from visual or nonverbal cues or status difference between the two (e.g., race, gender, age, voice tones, dress, shyness, gestures, disabilities). Murray and Harrison (2004), for example, argue that some of their potential participants - stroke survivors-were assumed not to be able or willing to take part in face-to-face interviews because of speech and mobility disabilities or self-consciousness about their appearance. Kim et al. (2003), too, explain that, among other things, e-mail may safeguard against possible loss of face among some people when they describe potentially sensitive events, experiences, or personal characteristics (e.g., difficult relationships with family, lack of English proficiency, racism, academic problems), thus allowing them to participate in research studies. In short, in many cases e-mail facilitates greater disclosure of personal information, offering further benefits to both the researcher and participants (Bowker \& Tuffin, 2004).

Another medium-related problem in e-mail interviewing is that it is always possible that some participants may not be as effective writers as they are speakers (Karchmer, 2001). As mentioned earlier, however, the opposite could be true, too. There could be some participants (and even interviewers) who do not or cannot express themselves as well in talking as they do in writing. Online communication could solve the latter problem because neither the participants nor the interviewers need to communicate orally or face-to-face.

Acknowledging that e-mail has strengths and weaknesses as a communication medium, researchers strive to maximize the richness of the tool by employing certain linguistic methods, such as the use of acronyms or abbreviations (e.g., LOL, laughing out loud; ROFL, rolling on the floor laughing) and emoticons (e.g., those little smiley faces), as well as underlining and capitalization (for emphasis), as a substitute for nonverbal cues (Walther, Anderson, \& Park, 1994). Because little is known about the number of e-mail users who are literate with these communication methods, it is 
important for researchers who use e-mail interviewing as a data collection method to instruct and encourage their participants to use such acronyms and emoticons. This will not only lessen some of the losses in nonverbal cues but it should also increase the depth of the data collected.

\section{Interview Questions}

As in face-to-face and telephone interactions, most e-mail interview-based studies use an interview schedule for data collection. Some researchers decompose the schedule into several sections and ask a certain number of questions at one time, whereas others send all primary interview questions in one e-mail message (see Table 1). Moreover, some researchers e-mail their questions only after securing permission from their participants. Others e-mail their interview questions along with the interview invitation and consent form so that potential participants will have a better idea of what would be involved in the interview process before any commitments are made.

The lack of a standard for conducting e-mail interviews is due to variations in the length of an interview schedule, the characteristics of the target population, and the experiences of the researchers in conducting qualitative e-mail interviews. With the exception of Meho and Tibbo (2003), no research report explicitly explained why one method was used but not another. In their case, a pretest was conducted to determine which method was best for their participants. The result indicated that all interview materials could be sent together in one e-mail, including the invitation for participation, background information about the researchers, consent form, instructions, and the interview schedule. This strategy may not be appropriate with other populations. The point here, however, is that pretests help determine the best method for each individual study or group of participants.

Meho and Tibbo (2003), as well as other researchers such as Curasi (2001) found that the interview guide containing the interview questions could be sent to informants via e-mail with the questions embedded in the e-mail message, rather than in an attached document. Research has shown that embedded questions result in significantly higher response rates (five times as much) than do those attached to an e-mail message (Dommeyer \& Moriarty, 2000). This is because the attached e-mail questions present too many obstacles to the potential respondent. Anyone responding to an attached e-mail survey must have: a strong interest in responding; the hardware and software that will enable him/her to download, read, and upload a foreign file; the knowledge of how to execute the various response steps; and a low fear of computer viruses. Absence of any one of these could result in a nonresponse. The embedded e-mail survey, despite its formatting limitations, can be answered and returned by the most unsophisticated of e-mail users and therefore can appeal to a broader audience (Dommeyer \& Moriarty).

A distinctive feature in e-mail interviewing is that it allows participants to take their time in answering questions and to take part in the interviews in a familiar environment (e.g., home or office), which may make them feel more relaxed expressing themselves and in responding when and how they feel comfortable (Kennedy, 2000; Lehu, 2004). Although this may generate rich and high quality data, it also means that the e-mailed questions must be much more selfexplanatory than those posed face-to-face, with a clear indication given of the responses required. Even when questions are pretested, because of lack of face-to-face or direct interaction in e-mail interviews, there is always room for miscommunication and misinterpretation. The inclusion of additional information may, however, function to narrow participants' interpretations and, thereby, constrain their responses. Therefore, managing this methodological dilemma requires meticulous attention to detail, with attempts to reduce ambiguity and improve specificity while avoiding the narrowing of participants' interpretations and constraint of their responses. According to Bowker and Tuffin (2004), restricting some of the ideas chosen for analysis will be inevitable, but it is very important and necessary to minimize participants' confusion and eventual frustration by specifying the meaning of interview questions. The following two examples from Meho and Tibbo's study (2003) demonstrate cases for which additional explanation of questions is needed:

Interview Question: Who and when do you usually ask
for help in locating research information? For what
kind(s) of help do you normally ask?
Participant Answer: I don't know what you mean here. I
usually hire a graduate student to do some basic legwork
for me in terms of hunting out the newest information on
whatever subject I am working on at the time.
Interview Question: What criteria do you employ when
assessing whether to follow up on materials not found in
your university library?
Participant Answer: Don't know what you mean by this.

The fact that these two questions were not clear to, or were misinterpreted by, only a very small fraction of the study participants $(3.3 \%)$ suggests that additional explanation or follow-up on misinterpreted questions be provided on an individual basis rather than to all participants. This should especially be the case when such questions are interpreted correctly by the majority of the study participants.

\section{Probes}

Probes or follow-up questions in interviews are generally used to elaborate and clarify participants' responses or to help elicit additional information and depth from informants. Unlike face-to-face and telephone interviews, e-mail interviews do not allow direct probing; it can be done only in follow-up e-mails, which can take place any time during the data collection and analysis periods.

The lack of direct probing in e-mail interviews may result in missing some important pieces of data, especially given that not all participants respond to follow-up questions, even if they were told to expect them. In Kennedy's study (2000), 
for example, 23 participants were initially involved; yet 3 stopped communicating after the first set of questions were answered and 3 did not supply enough information for analysis. In Karchmer's study (2001), 16 initially agreed to participate, but 3 discontinued correspondence within the first week of data collection. In Meho and Tibbo's (2001) study, 15 of the 60 study participants terminated the interview process and did not answer any of the follow-up questions. There are, however, cases in which all participants responded to follow-up probes, such as in Curasi's (2001) study.

As expected by researchers, the lack or loss of communication after the initial interview can be frustrating (Hodgson, 2004), but in none of the studies reviewed here was there discussion of this problem or an indication that this loss had any impact on the quality of data collected. In fact, although the lack of direct probing may result in the loss of some important pieces of information, on the other hand, it can play a major role in improving the quality of the e-mail interview data (Lehu, 2004). This is because the researcher is not limited to the probes that come to mind during the face-to-face interviews and because it gives participants ample time to think about their answers before sending them (Curasi, 2001). The benefits of indirect probing are further discussed in the following section.

\section{Data Quality}

According to Denscombe (2003, p. 51), the quality of responses gained through online research is much the same as responses produced by more traditional methods. The same conclusion was reached in several studies that compared, or conducted, both e-mail and face-to-face interviews (e.g., Curasi, 2001; Meho \& Tibbo, 2003; Murray, 2004; Murray \& Harrison, 2004). These studies found that participants interviewed via e-mail remained more focused on the interview questions and provided more reflectively dense accounts than their face-to-face counterparts. This is not to say that the quality of face-to-face interviews is lower, but rather to highlight the benefits of the e-mail interview, which was possibly aided by the ability of both the researchers and the interviewees to take the time to be more thoughtful and careful in their responses to, or communication with, each other than they would during natural conversation (Karchmer, 2001; Murray, 2004; Young et al., 1998).

Data quality, according to Curasi (2001), is dependent on who is being interviewed, who the interviewers are, and how skillful they are in online interviewing. She found, for example, that some e-mail interview participants provided very short and very precise responses to the questions posed. Others, however, discussed at length their feelings and experiences, sometimes in as much depth and detail as their face-to-face counterpart, especially when data from the initial questions are combined with those from follow-up questions. In other studies, data from face-to-face interviews did not reveal any information that was not already discovered via data from e-mail interviews (Meho \& Tibbo, 2003). Still other studies found that much of the information conveyed through electronic mail is information that would not be conveyed through another medium, such as sensitive and personal information-health, medical, political, and so on (Beck, 2005; Murray \& Sixsmith, 1998).

Overall, e-mail interviewing offers an opportunity to access, in an interactive manner, participants' thoughts, ideas, and memories in their own words. It allows the recording of many anecdotes that participants share to enhance the accounts of their experiences. It also allows participants to construct their own experiences with their own dialogue and interaction with the researcher. E-mail interviewing is additionally empowering to the participants because it essentially allows them to be in control of the flow of the interview (Bowker \& Tuffin, 2004), enabling them to answer at their convenience and in any manner they feel suitable (Kennedy, 2000). Levinson (1990) considers that the asynchronous electronic communication's capacity to provide opportunity for reflection and editing of messages before sending them contributes to the production of a closer fit between ideas, intentions, and their expression in writing. A summary of the advantages and disadvantages of e-mail interviewing, or challenges associated with it, is provided in Table 2.

\section{Guidelines for Conducting Effective E-Mail Interviews}

In addition to the findings discussed above, the studies reviewed or examined in this article and the personal experience of the author offer several suggestions for those considering the use of e-mail interviews in qualitative research. These suggestions are presented here in order to assist researchers in conducting more efficient and effective e-mail interviews, as well as to enable them to establish trustworthy results:

- Invitations: Solicit people for participation individually if possible rather than via a mailing list or message board. According to Dillman (2000), this technique shows potential participants that they are important, thereby encouraging them to participate.

- Subject line: Use an effective subject line for the first contact with the interviewees, such as Research Interview. This will avoid or reduce the likelihood of a request being deleted before it is read.

- Self-disclosure: Introduce yourself and provide brief information about your professional status/credentials. Then tell your interviewees how you acquired their e-mail addresses. This will help to establish trust. There is evidence that people will engage in more self-disclosure when they first become recipients of such self-disclosure from their interviewers (Moon, 2000).

- Interview request: State your request succinctly and professionally, as in "May Iinterview you for an article I am writing?"

- Be open about the research: Suspicion can exist when online researchers contact participants. One way to establish trust that creates rapport is to be as open as possible about the purposes and processes of the research. Outline the details of the project and specify the topic of the interview and the interview procedure, including information about 
Interviewers and participants

Cost

Time

Recruitment

Participation

Medium

effects

Data quality
Allows access to individuals often difficult or impossible to reach or interview face-to-face or via telephone

Allows access to diverse research subjects

Allows access to individuals regardless of their geographic location

Allows interviewing of individuals who do not or cannot express themselves as well in talking as they do in writing

Allows interviewing of individuals who prefer online interaction over face-to-face or telephone conversation

Eliminates expenses of calling and traveling

Eliminates expenses of transcribing

Decreases cost of recruiting large/geographically dispersed samples

Eliminates time required for transcribing

Eliminates the need to schedule appointments

Allows interviewing more than 1 participant at a time

Done via e-mail, listservs, message boards, discussion groups, and/or Web pages

Done by e-mail

Allows participants to take part in the interviews in a familiar environment (e.g., home or office)

Allows participants to take their time in answering questions

Allows participants to express their opinions and feelings more honestly (because of sense of anonymity)

Encourages self-disclosure

Eliminates interruption that takes place in face-to-face/telephone interviews

Eliminates transcription errors

Eliminates interviewer/interviewee effect resulting from visual and nonverbal cues or status difference between the two (e.g., race, gender, age, voice tones, dress, gestures, disabilities)

Cues and emotions can be conveyed through use of certain symbols or text

Allows participants to construct their own experiences with their own dialogue and interaction with the researcher

Facilitates a closer connection with interviewee's personal feelings, beliefs, and values

Data are more focused on the interview questions asked

Responses are more thought out before they are sent
Limited to individuals with access to the Internet

Requires skills in online communication from both interviewer and interviewees

Requires expertise in technology from both interviewer and interviewees

Can be high for participants

May take several days or weeks before an interview is complete

Invitations for participation may be deleted before they are read

High undeliverable rates (e.g., due to inactive e-mail addresses)

Some participants may drop out before interview is complete

Empowers participants, essentially allowing them to be in control of the flow of the interview

Does not allow direct probing

Requires that questions be more self-explanatory than those posed face-to-face or by telephone, to avoid miscommunication and misinterpretation

Loses visual and nonverbal cues due to inability to read facial expressions or body languages or hear the voice tones of each other

May narrow participants' interpretations and, thereby, constrain their responses

Requires meticulous attention to detail

Participants may lose focus

One-dimensional (based on text only)

In-depth information is not always easily obtainable follow-up exchanges. It is important that participants know what types of questions to expect, how much time is required for participation, and how many times they will be contacted. Made aware of this information, potential interviewees will likely make more informed decisions on whether to participate, which will assure better continuity in the interview process and in the quality of data collected.

- Incentives: Consider providing nontraditional incentives for people who will be willing to participate in a study. Meho and Tibbo (2003), for example, offered their study participants online bibliographic searches and personal citation searches (see Table 1). Promising participants a copy of the results may help encourage individuals to participate. Researchers should also communicate to potential participants the benefits of participation, such as the opportunity to gain perspectives on, and understanding of, their own ideas and opinions and those of their peers.

- Research ethics and informed consent: Emphasize the anonymity of the participants (e.g., by assuring them that all implicit and explicit links between their names and the data 
they provide will be removed). In addition, follow and communicate the standard procedures for the protection of human subjects to the participants, such as asking them to read an approved informed consent form before the interview takes place. Avoid overly elaborate assurances of anonymity and confidentiality because it may actually heighten rather than diminish respondents' concern, causing participants to be less willing to provide sensitive information (Singer \& Levine, 2003).

- Interview questions: Keep in mind that participants are not being interviewed face-to-face. So, as mentioned earlier, make sure that the questions to be asked are clear enough both to avoid misinterpretations and to motivate participants to delve deeper into the topic at hand. Also, determine whether there is a need to ask a certain number of questions at a time or ask all initial, important questions in the very first e-mail message - this largely depends on the nature of the study, the number of questions prepared, and the participants; these points can be verified by conducting pretests (Meho \& Tibbo, 2003; Young et al., 1998).

- Instructions: Along with the initial interview questions, include instructions to the participants on completing the interview. This might include how or where to place their answers; that the more detailed their responses the better; that there are no wrong or incorrect answers (in an effort to encourage spontaneity and reduce inhibitions); that they can use acronyms and symbols that communicate feelings, emotions, and the like; and that they should not worry about misspellings or grammatical errors.

- Deadlines and reminders: Indicate the due dates when inviting individuals to participate, but make them reasonable for the participants so that they have ample time to respond. Send reminders 1 week before the deadline, in case of no reply, to increase the response rate. When sending reminders, e-mail all important materials again (e.g., informed consent, interview schedule/questions, and so on) because some participants may feel shy about admitting that they deleted previously sent information. Limit the number of reminders to one or two; otherwise, it may be construed as pressure to continue participation.

- Follow-up questions: Be timely with follow-up questions, especially when clarifications, illustrations, explanations, or elaborations are needed. Check for messages from interviewees regularly and if necessary, summarize the interviewee's responses to previous questions and return the summary to the interviewee for verification. This will demonstrate understanding and concern for careful representation while allowing for clarification of misinterpretations (Young et al., 1998).

- Participants and data quality: Be very discriminating as to the sample interviewed. A highly committed or motivated participant can be very helpful, providing detailed and in-depth interviews. Conversely, potential informants who lack commitment to the project may not be worth the follow-up, extra energy, and the possible time delays they require (Curasi, 2001). Be alert for misunderstandings and attentive to changes in the tone of responses, symbols that are inconsistent with previous dialogue, and any other clues that might lead to questioning the credibility of a response. Be prepared to refocus the discussion on the interview topic(s). Online relationships that develop over longer time frames can become quite comfortable for the interviewee and there may be a tendency toward selfdisclosure beyond the scope of the interview topic(s). Do not overtly discourage this sharing; rather, subtly encourage responses related to the research topic (Young et al., 1998). Finally, reserve a few, or try to identify new, potential participants and use them as backup subjects. These may be needed if too many participants withdraw or if more data are needed.

- Survey methodology: Review the literature of, and learn how to carry out successful, survey research; it can be very useful in designing and conducting effective in-depth e-mail interviewing studies. Excellent sources on the topic include Dillman (2000), Groves, Dillman, Eltinge, and Little (2002), Groves et al. (2004), Presser et al. (2004), and Shaw and Davis (1996).

\section{Conclusion}

This article reviewed studies that used e-mail for conducting qualitative, in-depth interviews. It was found that e-mail interviewing offers unprecedented opportunities for qualitative research, providing access to millions of potential research participants who are otherwise inaccessible. The method can be employed quickly, conveniently, and inexpensively and can generate high-quality data when handled carefully. Although the method has a number of challenges, many of them were found to be easy to overcome, presenting scholars with a new technique for conducting efficient and effective qualitative research. While a mixed mode interviewing strategy should always be considered when possible, semi-structured e-mail interviewing can be a viable alternative to the face-to-face and telephone interviews, especially when time, financial constraints, or geographical boundaries are barriers to an investigation.

The use of e-mail to collect qualitative data will certainly expand as Internet access and use become more prevalent. ${ }^{4}$ Empirical studies addressing relevant methodological issues are few, and thus there is a need to explore more fully the conditions under which in-depth e-mail interviewing can be most effective, the factors that may influence its reliability, how the implementation of some techniques may improve response rate and quality of data, and how respondents react to e-mail-based interviews in comparison to telephone and face-to-face interviewing.

\section{Acknowledgments}

I would like to thank Blaise Cronin, Alice Robbin, and Debora Shaw for their valuable comments and suggestions.

\footnotetext{
${ }^{4}$ With an estimated 800 million Internet users worldwide (Internet World Stats, 2005) and thousands of scholars utilizing the ease of access to many of these users, online research has become a field in its own right, boasting a number of peer-reviewed journals (e.g., Social Research Updatehttp://www.soc.surrey.ac.uk/sru/—and Sociological Research Onlinehttp://www.socresonline.org.uk/home.html), a plethora of book titles, and an association that draws hundreds of researchers from across the globe to its annual conference (The Association of Internet Researchers-http:// www.aoir.org/).
} 


\section{References}

Beck, C.T. (2005). Benefits of participating in Internet interviews: Women helping women. Qualitative Health Research, 15, 411-422.

Birnbaum, M.H. (2004). Human research and data collection via the Internet. Annual Review of Psychology, 55, 803-832.

Bowker, N., \& Tuffin, K. (2004). Using the online medium for discursive research about people with disabilities. Social Science Computer Review, 22(2), 228-241.

Brownlow, C., \& O'Dell, L. (2002). Ethical issues for qualitative research in on-line communities. Disability \& Society, 17, 685-694.

Burton, L.J., \& Bruening, J.E. (2003). Technology and method intersect in the online focus group. Quest, 55, 315-327.

Chase, L., \& Alvarez, J. (2000). Internet research: The role of the focus group. Library \& Information Science Research, 22, 357-369.

Couper, M.P. (2000). Web surveys: A review of issues and approaches. Public Opinion Quarterly, 64, 464-494.

Curasi, C.F. (2001). A critical exploration of face-to-face interviewing vs. computer-mediated interviewing. International Journal of Market Research, 43(4), 361-375.

Denscombe, M. (2003). The good research guide. Maidenhead: Open University Press.

Denzin, N.K., \& Lincoln, Y.S. (2005). The sage handbook of qualitative research. (3rd ed.). London: Sage Publications.

Dijk, T.A.V. (1997). Discourse studies: A multidisciplinary introduction. Thousand Oaks, CA: Sage Publications.

Dillman, D.A. (2000). Mail and Internet surveys: The tailored design method (2nd ed.). New York: John Wiley \& Sons, Inc.

Dommeyer, C.J., \& Moriarty, E. (2000). Comparing two forms of an e-mail survey: Embedded vs. attached. International Journal of Market Research, 42(1), 39-50.

Ess, C. (2002). Ethical decision-making and Internet research: Recommendations from the AoIR Ethics Working Committee. Association of Internet Researchers (AoIR). Retrieved February 27, 2005, from http://www.aoir.org/reports/ethics.pdf

Eysenbach, G., \& Till, J.E. (November 10, 2001). Ethical issues in qualitative research on Internet communities. British Medical Journal, 323(7321), 1103-1105.

Fidel, R. (1993). Qualitative methods in information retrieval research. Library \& Information Science Research, 15(3), 219-247.

Foster, G. (1994). Fishing with the Net for research data. British Journal of Educational Technology, 25(2), 91-97.

Frankel, M.S., \& Siang, S. (1999). Ethical and legal aspects of human subjects research on the Internet. American Association for the Advancement of Science (AAAS). Retrieved February 27, 2005, from http://www. aaas.org/spp/sfrl/projects/intres/report.pdf

Frost, F. (1998). Electronic surveys: New methods of primary data collection. In European Marketing Academy, Proceedings of the 27th EMAC Conference: Marketing, Research and Practice (1998). Stockholm: EMAC.

Gaiser, T.J. (1997). Conducting on-line focus groups: A methodological discussion. Social Science Computer Review, 15(2), 135-144.

Groves, R.M., Dillman, D.A., Eltinge, J.L., \& Little, R.J.A. (2002). Survey nonresponse. New York: John Wiley \& Sons, Inc.

Groves, R.M., Fowler, F.J., Couper, M.P., Lepkowski, J.M., Singer, E., \& Tourangeau, R. (2004). Survey methodology. Hoboken, NJ: John Wiley $\&$ Sons, Inc.

Gubrium, J.F., \& Holstein, J.A. (2002). Handbook of interview research: Context \& method. Thousand Oaks, CA: Sage Publications.

Herring, S.C. (1996). Computer-mediated communication: Linguistic, social and cross-cultural perspectives. Philadelphia, PA: John Benjamin Publishing Company.

Herring, S.C. (2002). Computer-mediated communication on the Internet. Annual Review of Information Science and Technology, 36, 109-168.

Hodgson, S. (2004). Cutting through the silence: A sociological construction of self-injury. Sociological Inquiry, 74(2), 162-179.

Internet World Stats. (2005). Internet World Stats: Usage and population statistics. Retrieved May 25, 2005, from http://www.internetworldstats. $\mathrm{com} /$
Karchmer, R.A. (2001). The journey ahead: Thirteen teachers report how the Internet influences literacy and literacy instruction in their $\mathrm{K}-12$ classrooms. Reading Research Quarterly, 36(4), 442-466.

Kennedy, T.L.M. (2000). An exploratory study of feminist experiences in cyberspace. CyberPsychology \& Behavior, 3(5), 707-719.

Kim, B.S.K., Brenner, B.R., Liang, C.T.H., \& Asay, P.A. (2003). A qualitative study of adaptation experiences of 1.5-generation Asian Americans. Cultural Diversity \& Ethnic Minority Psychology, 9(2), 156-170.

Knapp, M.L., \& Daly, J.A. (2002). Handbook of interpersonal communication (3rd ed.). Thousand Oaks, CA: Sage Publications.

Kraut, R., Olson, J., Banaji, M., Bruckman, A., Cohen, J., \& Couper, M. (2004). Psychological research online: Report of board of scientific affairs' advisory group on the conduct of research on the Internet. American Psychologist, 59(2), 105-117.

Krippendorff, K. (2004). Content analysis: An introduction to its methodology (2nd ed.). Thousand Oaks, CA: Sage Publications.

Kvale, S. (1996). Interviews: An introduction to qualitative research interviewing. Thousand Oaks, CA: Sage Publications.

Lehu, J-M. (2004). Back to life! Why brands grow old and sometimes die and what managers then do: An exploratory qualitative research put into the French context. Journal of Marketing Communications, 10, 133-152.

Levinson, P. (1990). Computer conferencing in the context of the evolution of media. In L.M. Harasim (Ed.), Online education: Perspectives on a new environment (pp. 3-14). NY: Praeger.

Madge, C., \& O'Connor, H. (2002). On-line with e-mums: Exploring the Internet as a medium for research. Area, 34(1), 92-102.

Madge, C., \& O'Connor, H. (2004). Online methods in geography educational research. Journal of Geography in Higher Education, 28(1), 143-152.

Mann, C., \& Stewart, F. (2000). Internet communication and qualitative research: A handbook for researching online. London: Sage Publications.

Matheson, K. (1992). Women and computer technology: Communicating for herself. In M. Lea (Ed.), Contexts of computer-mediated communication (pp. 66-88). Hemel Hempstead: Harvester Wheatsheaf.

Meho, L.I. (2001). The information-seeking behavior of social science faculty studying stateless nations. Unpublished doctoral dissertation, University of North Carolina at Chapel Hill.

Meho, L.I., \& Tibbo, H.R. (2003). Modeling the information-seeking behavior of social scientists: Ellis's study revisited. Journal of the American Society for Information Science and Technology, 54(6), 570-587.

Miles, M.B., \& Huberman, A.M. (1994). Qualitative data analysis: An expanded sourcebook. Thousand Oaks, CA: Sage Publications.

Moon, Y. (2000). Intimate exchanges: Using computers to elicit selfdisclosure from consumers. Journal of Consumer Research, 27, 323-339.

Murray, C.D. (2004). An interpretive phenomenological analysis of the embodiment of artificial limbs. Disability and Rehabilitation, 26(16), 963-973.

Murray, C.D., \& Harrison, B. (2004). The meaning and experience of being a stroke survivor: An interpretive phenomenological analysis. Disability and Rehabilitation, 26(13), 808-816.

Murray, C.D., \& Sixsmith, J. (1998). E-mail: A qualitative research medium for interviewing? International Journal of Social Research Methodology, $1(2), 103-121$.

Murray, P.J. (1995). Research from cyberspace: Interviewing nurses by e-mail. Health Informatics, 1(2), 73-76.

Murray, P.J. (1996). Nurses' computer-mediated communications on NURSENET: A case study. Computers in Nursing, 14(4), 227-234.

Olivero, N., \& Lunt, P. (2004). Privacy versus willingness to disclose in e-commerce exchanges: The effect of risk awareness on the relative role of trust and control. Journal of Economic Psychology, 25(2), 243-262.

Oppermann, M. (1995). E-mail surveys: Potentials and pitfalls. Marketing Research, 7(3), 29-33.

Panteli, N. (2002). Richness, power cues and email text. Information \& Management, 40(2), 75-86.

Patton, M.Q. (2002). Qualitative research and evaluation methods (3rd ed.). Thousand Oaks, CA: Sage Publications.

Persichitte, K.A., Young, S., \& Tharp, D.D. (1997). Conducting research on the Internet: Strategies for electronic interviewing. In Proceedings of Selected Research and Development Presentations at the 1997 National Convention of the Association for Educational Communications and 
Technology (19th, Albuquerque, NM, February 14-18, 1997). Washington, DC: Association for Educational Communications and Technology.

Pettigrew K.E., Fidel, R., \& Bruce, H. (2001). Conceptual frameworks in information behavior. Annual Review of Information Science and Technology, 35, 43-78.

Pittenger D.J. (2003). Internet research: An opportunity to revisit classic ethical problems in behavioral research. Ethics \& Behavior, 13(1), 45-60.

Presser, S., Couper, M.P., Lessler, J.T., Martin, E., Martin, J., Rothgeb, J.M., et al. (2004). Methods for testing and evaluating survey questions. Public Opinion Quarterly, 68(1), 109-130.

Robert, L.P., \& Dennis, A.R. (2005). Paradox of richness: A cognitive model of media choice. IEEE Transactions on Professional Communication, 48(1), 10-21.

Schiffrin, D., Tannen, D., \& Hamilton, H.E. (2001). The handbook of discourse analysis. Malden, MA: Blackwell Publishers.

Schneider, S.J., Kerwin, J., Frechtling, J., \& Vivari, B.A. (2002). Characteristics of the discussion in online and face to face focus groups. Social Science Computer Review, 20(1), 31-42.

Selwyn, N., \& Robson, K. (1998). Using e-mail as a research tool. Social Research Update, 21. Retrieved from http://www.soc.surrey.ac.uk/sru/ SRU21.html

Sharf, B.F. (1999). Beyond netiquette: The ethics of doing naturalistic discourse research on the Internet. In Steve Jones (Ed.), Doing Internet research: Critical issues and methods for examining the Net (pp. 243-256). Thousand Oaks, CA: Sage Publications.

Shaw, D., \& Davis, C.H. (1996). Modern Language Association: Electronic and paper surveys of computer-based tool use. Journal of the American Society for Information Science, 47(12), 932-940.

Singer, E., \& Levine F.J. (2003). Protection of human subjects of research: Recent developments and future prospects for the social sciences. Public Opinion Quarterly, 67(1), 148-164.
Strauss, A.L., \& Corbin, J.M. (1998). Basics of qualitative research: Techniques and procedures for developing grounded theory. Thousand Oaks: Sage Publications.

Taylor, S.J., \& Bogdan, R. (1998). Introduction to qualitative research methods: A guidebook and resource (3rd ed.). New York: John Wiley \& Sons, Inc.

Thurlow, C., Tomic, A., \& Lengel, L.B. (2004). Computer mediated communication: Social interaction and the Internet. Thousand Oaks, CA: Sage Publications.

Tidwell, L.C., \& Walther, J.B. (2002). Computer-mediated communication effects on disclosure, impressions, and interpersonal evaluations: Getting to know one another a bit at a time. Human Communication Research, 28(3), 317-348.

Tourangeau, R. (2004). Survey research and societal change. Annual Review of Psychology, 55, 775-801.

Underhill, C., \& Olmsted, M.G. (2003). An experimental comparison of computer-mediated and face-to-face focus groups. Social Science Computer Review, 21(4), 506-512.

Walther, J.B. (1996). Computer-mediated communication: Impersonal, interpersonal, and hyperpersonal interaction. Communication Research, $23,3-43$.

Walther, J.B., Anderson, J.F., \& Park, D.W. (1994). Interpersonal effects in computer-mediated communication: A meta-analysis of social and antisocial communication. Communication Research, 21, 460-487.

Wang, P. (1999). Methodologies and methods for user behavioral research. Annual Review of Information Science and Technology, 34, 53-99.

Weber, R.P. (1990). Basic content analysis (2nd ed.). Newbury Park, CA: Sage Publications.

Young, S., Persichitte, K.A., \& Tharp, D.D. (1998). Electronic mail interviews: Guidelines for conducting research. International Journal of Educational Telecommunications, 4(4), 291-299. 